\title{
Chemical Peels: Deep, Medium, and Light
}

\author{
Sidney J. Starkman, MD ${ }^{1}$ Devinder S. Mangat, MD, FACS ${ }^{1}$ \\ ${ }^{1}$ Mangat, Holzapfel \& Lied Plastic Surgery, Cincinnati, Ohio \\ Address for correspondence Devinder S. Mangat, MD, Mangat, \\ Holzapfel \& Lied Plastic Surgery, 8044 Montgomery Rd, Suite 230 \\ Facial Plast Surg 2019;35:239-247. \\ Cincinnati, OH 45236 (e-mail: devindermangat5@gmail.com).
}

\begin{abstract}
Keywords

- chemical peel

- chemoexfoliation

- phenol-croton oil peel

- skin resurfacing

Chemical peels, laser resurfacing, and dermabrasion all offer unique options for skin resurfacing for rhytids and dyschromias. Laser resurfacing has developed over the previous decades, but it is the chemical peel against which all modern forms of skin resurfacing are measured. Phenol-croton oil peels have been modernized and depend on croton oil concentration to minimize risks. Complications associated with skin resurfacing are uncommon with proper technique and postoperative management.
\end{abstract}

There has been a boom in skin care products, medical devices, and skin rejuvenation therapies over the past few decades. This is likely because of modern medicine increasing both the average lifespan and quality of life in the general population. Due to this development, there has been a greater demand for treatment of age-related skin changes. As many new over-thecounter options in skin rejuvenation are increasingly being used by patients, they are now more prepared for medical grade chemical peels for more potent and lasting results. The different variations of chemoexfoliation have been used for rhytids, actinic damage, lentigines, and dyschromias.

The goal of the following section is to describe the most recent knowledge about chemical peeling and to expose the previously accepted yet incorrect dogmas. Chemical peeling, when practiced with knowledge and good technique, can yield excellent results in skin rejuvenation.

\section{Patient Selection}

As with any patient encounter, the initial consultation provides an opportunity to thoroughly assess the patient's history and physical examination. The patient must both be a physical candidate for a chemical peel and have appropriate expectations for their postpeel results. For non-Caucasian patients, in particular African-American patients, a personal and family history of keloid and hypertrophic scar formation should caution the practitioner from aggressive resurfacing. Skin-related changes such as rhytids and photodamage must be distinguished from other changes such as volume loss or jowling. Ideally, a chemical peel patient will have blue eyes, fair skin, and shallow rhytids. However, the majority of chemical peel patients will not fit this exact description. Most commonly, the Fitzpatrick scale is used to help define a patient's skin type ( - Table $\mathbf{1}$ ).

Patients can also be rated by their skin type, texture, complexion, photoaging, and so on using categorizing schemes such as the one by Glogau ( - Table 2 ).

The medical history of the patient must be reviewed before any chemical peeling occurs. Relative contraindications for any resurfacing procedure include smoking, diabetes, active or frequent herpes simplex virus infections, cutaneous radiation history, hypertrophic scarring, or keloid history. Photosensitizing drugs, exogenous estrogen, and birth control pills should be avoided because of the increased risk of hyperpigmentation. Patients should also be warned not to have plans to become pregnant within 6 months after chemical peeling due to elevated estrogen levels of pregnancy. ${ }^{1,2}$

Smoking and sun exposure should always be addressed in the planning stages. Chemical peels on the faces of chronic smokers can lead to poor tissue healing due to the microvascular damage from smoking. It is recommended that smokers stop 1 month before the peel and continue abstinence for at least 6 months afterward. Likewise, it should be recommended to patients that for 3 months, prolonged sun exposure should be avoided after the peel. If this is unacceptable to the patient, other options besides chemical peeling should be explored.

An absolute contraindication to chemical peeling, or any facial resurfacing, is recent use of isotretinoin (Accutane). Isotretinoin prevents reepithelialization from hair follicles and sebaceous glands, and chemical peeling relies primarily
Issue Theme Anything But the Knife: Nonsurgical Facial Plastic Surgery; Guest Editors: Deborah Watson, MD, FACS, and David B. Hom, MD, FACS
Copyright (C) 2019 by Thieme Medical Publishers, Inc., 333 Seventh Avenue, New York, NY 10001, USA. Tel: +1(212) 584-4662.
DOI https://doi.org/ 10.1055/s-0039-1688944. ISSN 0736-6825. 
Table 1 Fitzpatrick skin type scale

\begin{tabular}{|l|l|l|}
\hline Skin type & Skin color & Characteristics \\
\hline I & White; very fair; red or blond hair; blue eyes; freckles & Always burns; never tans \\
\hline II & White; fair; red or blond hair; blue, hazel or green eyes & Usually burns, tans with difficulty \\
\hline III & Cream white; fair with any eye or hair color; very common & Sometimes mild burn, gradually tans \\
\hline IV & Brown; typical Mediterranean Caucasian skin & Rarely burns, tans with ease \\
\hline V & Dark brown; mid-eastern skin types & Very rarely burns, tans very easily \\
\hline VI & Black & Never burns, tans very easily \\
\hline
\end{tabular}

Table 2 Glogau skin classification scale

\begin{tabular}{|l|l|l|l|}
\hline Group I (mild) & Group II (moderate) & Group III (advanced) & Group IV (severe) \\
\hline No keratoses & $\begin{array}{l}\text { Early actinic keratoses: } \\
\text { slight yellow skin } \\
\text { discoloration }\end{array}$ & $\begin{array}{l}\text { Actinic keratoses: obvious } \\
\text { yellow skin discoloration } \\
\text { with telangiectasias }\end{array}$ & Actinic keratoses and skin cancers \\
\hline Little wrinkling & $\begin{array}{l}\text { Early wrinkling: } \\
\text { parallel skin lines }\end{array}$ & Wrinkling present at rest & $\begin{array}{l}\text { Wrinkling: much cutis laxa of actinic, } \\
\text { gravitational, and dynamic origin }\end{array}$ \\
\hline No scarring & Mild scarring & Moderate acne scarring & Severe acne scarring \\
\hline Little or no makeup & Little makeup & Wears makeup always & Wears heavy layers of makeup \\
\hline
\end{tabular}

upon this reepithelialization for healing. The most current recommendations are to stop isotretinoin for 12 to 24 months before the peel.

Finally, the patient and the practitioner must have agreed upon the reasonable expectations of the peel. The patient's axillary skin can represent the final result of the chemical peel as long as this region has not previously received excessive sun damage. ${ }^{3}$

\section{Preparation}

After the patient selection and planning is completed, one must address patient concerns, establish realistic expectations, and, ultimately, decide on the appropriateness to pursue the resurfacing procedure. Sunscreens should be started 3 months beforehand to prevent prepeel tanning or sunburns. Part of the purpose of this is to decrease the melanocyte activity before the peel. Topical tretinoin (Retin-A; Bausch Health Companies Inc.) is recommended for 6 to 12 weeks before the peel. The topical tretinoin has been shown to have a synergistic effect with trichloroacetic acid (TCA) peels and can sustain the effects of these peels. ${ }^{4-6}$ Tretinoin leads to exfoliation of stratum corneum and increased melanin distribution and aids in proper penetration of the peel solution. It contributes to a thickened and uniform epidermis, which aids in uniform application of the peeling agent.

Up to 6 weeks before resurfacing, patients can be started on topical therapies to increase the rate of epithelialization, stabilize melanocyte activity, and reduce local inflammation. The dosing ranges from 0.025 to $0.1 \%$; however, no literature has shown improved results with the higher dosing. Patients should be counseled of the possible side effects of tretinoin, such as erythema, flakiness, or skin irritation. If this was to occur, the dose can be reduced or the medication can be discontinued entirely.
Another beneficial drug in the preparation of chemical peel patients is hydroquinone. Hydroquinone is mostly used in patients with dyschromias and lentigines or patients with Fitzpatrick skin types III, IV, V, and VI due to the elevated risks of postpeel postinflammatory hyperpigmentation (PIH). The mechanism of hydroquinone is to block the conversion of tyrosine to L-Dopa by tyrosinase, thereby decreasing melanin production. In applicable patients, hydroquinone in a concentration of 4 to $8 \%$ should be started 4 to 6 weeks before chemical peeling. The authors use a topical preparation of $0.025 \%$ tretinoin, $8 \%$ hydroquinone, and $1 \%$ hydrocortisone in a moisturizing cream base. Similar to tretinoin, hydroquinone should be restarted after the peel once the patient's skin is ready to tolerate it. Topical therapy is stopped 3 to 4 days before the procedure but may be restarted 1 week after the procedure. Oral antiherpetic prophylaxis is initiated 3 days before resurfacing and continued 5 to 7 days until reepithelialization is complete.

The first line of resistance to bacterial infection is the skin, and resurfacing procedures can reduce this barrier. This can lead to infections by cutaneous bacterial flora such as staphylococcal or streptococcal species. Appropriate antibacterial coverage should begin before the peel as prophylaxis. The senior author (D.S.M.) uses cephalexin, $250 \mathrm{mg}$ four times a day, 1 day before the peel and continues it for 7 days in the postoperative period. In patients who are B-lactam sensitive, erythromycin, $250 \mathrm{mg}$ four times a day, can be used. To maintain a uniform depth of the peeling agent, it is advisable to recommend avoiding microdermabrasion, waxing, or electrolysis for 3 to 4 weeks before peeling.

\section{Superficial Peels}

The workhorse peel for plastic surgeons, dermatologists, and aestheticians has been the $\alpha$-hydroxy acids (AHAs). AHAs are natural fruit, carboxylic acids, with the most common AHA 
being glycolic acid or 2-hydroxyethanoic acid. Glycolic acid is derived from sugarcane and is generally used in a concentration of 20 to $70 \%{ }^{6,7}$ Salicylic acid, in concentrations of 20 to $30 \%$, is also used in superficial chemical peels.

$\alpha$-hydroxy acids penetrate through the epidermis and into the most superficial layer of the papillary dermis. They work by diminishing the cohesion between the keratinocytes of the stratum granulosum. This leads to the sloughing of the abnormal cells and thins the stratum corneum. These benefits generally last for 2 to 3 weeks.

A false sense of security should be avoided with AHAs. Glycolic acid can have a persistent effect and penetrate deeply if not neutralized with water. If glycolic remains unneutralized, the deeper penetration can lead to healing problems, crusting, and scarring. The anticipated end of a glycolic acid peel procedure should be erythema and light peeling of the epidermis. ${ }^{8}$

Jessner's solution (14 $\mathrm{g}$ of salicylic acid, $14 \mathrm{~g}$ of lactic acid, $14 \mathrm{~g}$ of resorcinol in $100 \mathrm{~mL}$ of ethanol) is a superficial peeling agent with a high safety profile. The number of coats applied determines the depth of penetration. If one to three coats are applied, exfoliation of the stratum corneum occurs. If 5 to 10 coats are applied, the depth of penetration continues down to the basal layer.

\section{Deep Peels}

The deep peel treats the deep rhytids of the lateral canthal and perioral regions by penetrating down to the reticular dermis. While the papillary dermis is thought to heal through reorganization, the reticular dermis is thought to heal through scarring. ${ }^{9}$ With deep chemical peeling, the risk of scarring is higher and additional recovery time is necessary.

The workhorse deep chemical peel agent was the BakerGordon formulation; however, this lost some popularity as TCA peels and $\mathrm{CO}_{2}$ laser surfacing rose into the field. The BakerGordon peel did offer arguably unparalleled treatment for deep facial rhytids, but the irreversible hypopigmentation risk and the cardiac/renal toxicity limited its applicability.

Another option for deep peels is the 50\% TCA peel; however, a significant percentage of patients develop scarring with this formulation. This catastrophic complication halted the application of this strength of TCA solution.

Notwithstanding the risks of the Baker-Gordon peel, it does have a role in the correct scenario. It is a very effective peeling option in patients with deepened rhytids in distinct facial subunits with Fitzpatrick skin types I and II. Nonetheless, a high level of technique and caution must be used to avoid overly deep peeling with this solution. A uniform application of peeling solution with attention to the developing frost will help prevent potential complications.

\section{Medium-Depth Peels}

The modern-day chemical peel discussions center around the medium-depth peeling agents. Medium-depth peels penetrate through the epidermis and the papillary dermis and cause some inflammation in the upper reticular dermis. Traditionally, the standard medium peel has been the 35\% TCA peel. It does not have systemic toxicities, and it is very easy to store as it does not require refrigeration in its crystalline form. However, the risk of scarring with TCA is much higher than with phenolbased peeling solutions. TCA concentrations of $50 \%$ or greater greatly increase the possibility of scarring. ${ }^{10}$ There have been no additional techniques that have proven beneficial in reducing this risk of scarring. ${ }^{11}$

Providers have been combining 35\% TCA solutions with other less potent agents to achieve medium-depth peels without the risks of scarring. The depth of penetration of the 35\% TCA peel solution is enhanced by an additional solution being first applied as an epidermolytic. This technique was first described by Brody using $\mathrm{CO}_{2}$ ice and acetone to create an epidermal break for the TCA solution that follows. ${ }^{12}$

Monheit described another combination peel: Jessner's solution followed by $35 \%$ TCA. Jessner's plays the role of penetrating the epidermis, and TCA is then applied once the Jessner's solution has dried. ${ }^{13}$ Frosting does not occur immediately, unlike with phenol peels, and 3 to 4 minutes must be allowed for the full frost to form. Once the frost occurs, additional coats can be applied to reach the desired depth of peel. Care is taken with additional applications of TCA, as this has a cumulative effect and leads to a deeper peel. ${ }^{14}$ This can increase the chances of hypopigmentation and scarring.

There are also combination peels made out of glycolic acid and 35\% TCA, as described by Coleman and Futrell. His histological examinations demonstrated that it penetrated slightly deeper than Jessner-TCA combination peels. ${ }^{15}$

Brody researched the complications of these three combination peels and found that their risks of scarring were less than $1 \%$. This scarring risk placed them on par with other skin resurfacing modalities such as phenol-croton oil peels or $\mathrm{CO}_{2}$ laser resurfacing.

\section{Modified Phenol-Croton Oil Peels}

The all-or-none qualities of the Baker-Gordon phenol-croton oil peel has led to TCA peel solutions taking a prominent role in modern-day chemical peel practices. The literature on deep chemical peels began in the 1950s and 1960s when plastic surgeons first adopted the phenol-croton oil solutions. Litton was the first to present these formulas to the American Society of Plastic and Reconstructive Surgery in the late 1950s. Soon afterward, the classic formula was credited to Baker in the early 1960s. ${ }^{16}$

Around this time in the 1960s, Adolph Brown wrote extensively about phenol-croton oil peels. He presented three doctrines of phenol peeling. First, increasing the concentration of phenol (80-90\%) would work to prevent further penetration by creating an immediate keratocoagulation. Second, adding a saponin to the solution would increase the depth of penetration. Third, the role of croton oil was merely to buffer the solution. This led to many believing that phenol was the sole active ingredient within the Baker formula. This resulted in the belief that phenol in lower concentrations was more dangerous because of deeper penetration and that croton oil had no role in the depth of peel. These beliefs lasted until they were questioned by Gregory Hetter in the 1990s. 
Hetter's experiments refuted the previously described dogmas of the mid-20th century. A solution of $18 \%$ phenol without croton oil demonstrated minimal postpeel effect. With a $35 \%$ phenol solution, mild keratolysis occurred with no dermal effect. It was only with an $88 \%$ phenol solution that a papillary dermal effect took place. More substantial postpeel effects were noticed once croton oil was added to the phenol solution. Additionally, varying croton oil concentrations had different results. A $0.7 \%$ croton oil concentration solution required a 7 -day recovery period, whereas a $2.1 \%$ croton oil concentration required an 11-day recovery period. Hetter thus postulated that higher concentrations of phenol (88\%) without septisol peel more deeply than lower concentrations (50 and $35 \%)$. He concluded that increasing the concentrations of croton oil in phenol formulations results in deeper peels.

Hetter also realized that the healing times would be shortened by diluting the concentration of croton oil in these formulas, signifying a shallower depth of penetration. He expanded on this by claiming that the concentration of phenol, in fact, had little to do with the depth of penetration. Obagi was the first to suggest that different concentrations of these formulas should be applied to the discrete subunits of the face. Hetter used this postulation to apply varying concentrations of croton oil to the facial subunits ( $\mathbf{- F i g . 1}$ ). He found that the temple and lateral brow could only withstand concentrations up to $0.6 \%$, the cheeks and forehead could only tolerate concentrations up to $0.8 \%$, and the perioral region could tolerate croton oil concentrations up to $1.6 \%$ before the risk of complications rose. Lastly, Hetter felt that $1 \%$ croton oil solutions were the upper threshold for safe use to avoid serious risk of hypopigmentation.

Hetter first created his formulations using phenol at 33\% to carry croton oil at one $\operatorname{drop}(0.35 \%)$, two drops ( $0.7 \%)$, and three drops (1.1\%) of concentrations. However, he soon switched to a more standardized system of measurement rather than relying on a dropper, which is naturally unreliable. He converted one drop into $1 \mathrm{~cm}^{3}$ and used this conversion to make a stock solution of $0.04 \mathrm{~mL}$ of croton oil per $1 \mathrm{~mL}$ of phenol. From this, he could make different croton oil formulations of $0.4,0.8,1.2$, and $1.6 \%$ in a constant phenol concentration (-Table 3 ).

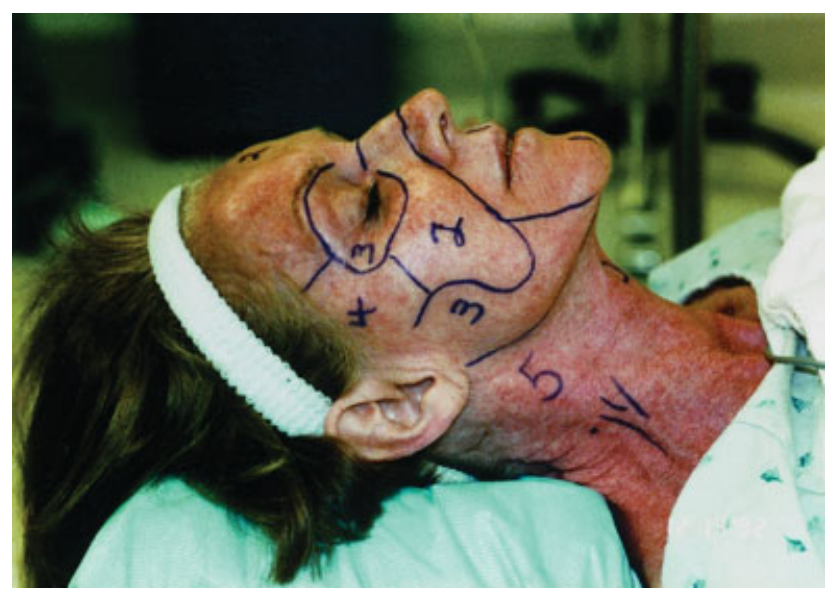

Fig. 1 Facial subunit markings demonstrating various concentrations of peel solution, as described by Hetter.
Table 3 Hetter peel formulations (stock solution $=24 \mathrm{~mL}+1$ $\mathrm{mL}$ croton oil [ $4 \%$ croton oil])

\begin{tabular}{|l|l|l|l|l|l|}
\hline Croton oil \% & $\mathbf{0 . 2 \%}$ & $\mathbf{0 . 4 \%}$ & $\mathbf{0 . 8 \%}$ & $\mathbf{1 . 2 \%}$ & $\mathbf{1 . 6 \%}$ \\
\hline $\begin{array}{l}\text { Distilled } \\
\text { water }\end{array}$ & $5.5 \mathrm{~mL}$ & $5.5 \mathrm{~mL}$ & $5.5 \mathrm{~mL}$ & $5.5 \mathrm{~mL}$ & $5.5 \mathrm{~mL}$ \\
\hline Septisol & $0.5 \mathrm{~mL}$ & $0.5 \mathrm{~mL}$ & $0.5 \mathrm{~mL}$ & $0.5 \mathrm{~mL}$ & $0.5 \mathrm{~mL}$ \\
\hline $\begin{array}{l}\text { USP } \\
\text { phenol } 88 \%\end{array}$ & $3.5 \mathrm{~mL}$ & $3.0 \mathrm{~mL}$ & $2.0 \mathrm{~mL}$ & $1.0 \mathrm{~mL}$ & $0 \mathrm{~mL}$ \\
\hline $\begin{array}{l}\text { Stock } \\
\text { solution } \\
\text { containing } \\
\text { phenol and } \\
\text { croton oil }\end{array}$ & $0.5 \mathrm{~mL}$ & $1.0 \mathrm{~mL}$ & $2.0 \mathrm{~mL}$ & $3.0 \mathrm{~mL}$ & $4.0 \mathrm{~mL}$ \\
\hline Total & $10 \mathrm{~mL}$ & $10 \mathrm{~mL}$ & $10 \mathrm{~mL}$ & $10 \mathrm{~mL}$ & $10 \mathrm{~mL}$ \\
\hline
\end{tabular}

\section{Technique}

The skin must be sufficiently prepared before applying the peeling solution. This starts with a vigorous cleaning with acne wash or septisol the evening before and the morning of the chemical peel. Preoperative oral sedation, $10 \mathrm{mg}$ of diazepam and $100 \mathrm{mg}$ of Dramamine, helps to relieve patient anxiety. The antihistamine serves to reduce oral secretions and protect the patient's airway during the sedation. As the patient will have had nothing to drink since the previous night, intravenous fluids should be started prior to bringing the patient to the procedure room.

Preoperative marking consists of marking the patient's submandibular shadow while in the upright position. This helps to prevent noticeable delineation between the peeled and unpeeled area of the neck. The patient is then placed in the supine position.

Supraorbital, infraorbital, and mental nerve blocks are performed with 50:50 mixture of $2 \%$ lidocaine and $0.5 \%$ bupivacaine. Local anesthetic is also applied through a field block over the areas to be peeled. Epinephrine will slow the clearance of the phenol and is thus avoided. The face is meticulously degreased with an acetone-soaked gauze. Any remaining skin oil on the patient's face will impair the uniform application of the peeling agent. Some authors like Obagi and Hetter suggest using a wrung-out 2-inch by 2-inch gauze for the application of the peeling agent. However, However, the senior author (D.S.M.) feels that using a wide cotton-tipped applicator allows for superior control of application.

As opposed to TCA where the frost can take 3 to 4 minutes to occur, with phenol-based chemical peels, the frosting occurs almost immediately. This means that the uniformity of depth, and need for reapplication, is almost immediately obvious to the provider in a phenol-based peel. Mediumdepth peels should result in a level II to III frost (-Fig. 2). ${ }^{17}$

- Level I: erythema with stringy or blotchy frosting.

- Level II: white coat with erythema showing through (it should be used for eyelids and areas of bony prominences, such as zygomatic arch, malar region, and chin, which all have a higher rate of scarring).

- Level III: solid white frost with little or no background erythema. 


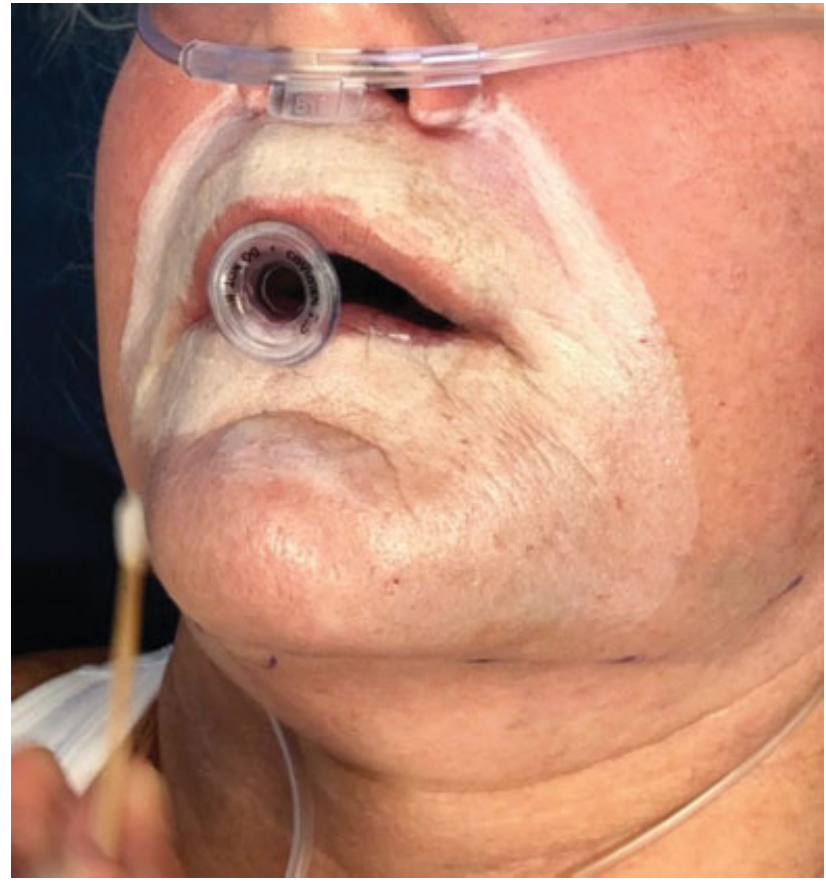

Fig. 2 A level III frost after application of phenol-croton oil solution, with a white frost over the treated skin.

The facial subunits are divided by the severity of lentigines, rhytids, and photodamage, as well as skin thickness. The senior author (D.S.M.) uses $0.8 \%$ croton oil Hetter solution in areas of thicker skin and deeper rhytids (Glogau III and IV), such as the glabella and perioral regions. Intermediate areas (Glogau II and III) are treated with $0.4 \%$ croton oil Hetter solution. An $89 \%$ phenol solution is used for feathering along the borders of the peeled areas to achieve an even postpeel result. A classic Baker formula can be used in patients who are Fitzpatrick types I or II and have severe Glogau IV rhytids in the upper lip.

Between each facial subunit, 10 to 15 minutes are allowed for adequate clearance of the phenol. The Hetter solution requires less clearance time due to lower phenol compared with the Baker-Gordon peel. The entire face can be peeled over 30 to 60 minutes. In case a minor supraventricular arrhythmia occurs, the peel should be paused until the patient returns to normal sinus rhythm.

The peel should continue up to and even into the hairline when applying the peeling solution to the forehead and temporal regions. Phenol and croton oil will not affect the pigment of the hair follicles. Additionally, the peel should continue just over the vermilion border in the perioral area. This is because the margin of each peeled region will have a distinct line of reactive hyperemia. These lines of hyperemia should be included and peeled when peeling adjacent facial subunits to prevent any resultant lines of demarcation. The skin can be stretched taut for deep wrinkles of the perioral region to evenly apply the peel to these rhytids. Unlike with glycolic acid peels, phenol-croton oil peels do not need to be neutralized because of the completed reaction as demonstrated by the frost.

An area to exercise caution is in the lower eyelid region. The phenol-croton oil peel should be applied to within $3 \mathrm{~mm}$ of the lower eyelid margin and then stopped. The sedated patient can develop tearing during the peeling procedure, and these tears should be wiped away to prevent the peeling solution from tracking up along the tear into the eye. The upper eyelid lacks the sebaceous glands that are necessary for reepithelialization following a peel, and therefore the peel solution is not applied to this area.

The patient can experience an immediate burning sensation if there were any areas of inadequate local anesthesia. In this case, the sensation will last for approximately 15 to 30 seconds. The burning sensation can then return approximately 30 minutes later and can last for the remainder of the procedure day. The bupivacaine in the local anesthetic blocks should provide anesthesia for hours following the peel. This is a part of the reason why it is critical to apply comprehensive local anesthetic blocks to the peeled areas.

\section{Postoperative Period}

A thick coat of emollient is applied to all of the peeled skin areas once the last area of frosting dissipates and only erythema remains. These emollients are not occlusive and therefore do not affect the depth of the peel. The patient is instructed to maintain a steady coat of this emollient over their entire peeled region by reapplying four to five times per day or as often as needed. This is continued for the duration of the postpeel period until fresh skin is visible and the area has fully peeled.

The healing process consists of four stages. The first stage occurs over the first 12 hours and consists of facial inflammation. The epidermis becomes leathery and begins to separate from the dermis. The underlying treated dermal layer will become necrotic and being to slough. The applied emollient helps in removing this necrotic skin from the underlying tissues. The second stage is desquamation, which occurs over the next 3 to 7 days (-Fig. 3). This exposed the underlying erythematous dermis. The third stage is reepithelialization, which partially coincides with desquamation and occurs between days 2 and 10 following the peel. Reepithelialization will be demonstrated by the changes in dermal color from bright red to a lighter shade of pink. The final stage is fibroplasia and occurs toward the end of the first week and continues for 12 to 16 weeks following the peel. This final period is when the full benefits of the chemical peel become apparent (-Figs. 4-11). During this time, the skin will undergo new collagen formation, reorganization of the collagen, and neoangiogenesis.

The patient is instructed to avoid any direct, prolonged sun exposure for 12 weeks following the peel. During this period, the skin is vulnerable to ultraviolet light exposure and is at a risk of resultant hyperpigmentation. The senior author (D.S.M.) also recommends avoidance of sunscreens for the first 6 weeks. Many chemical sunscreens include the ingredient para-aminobenzoic acid, which can cause erythema, irritation, and induration of the healing skin. It is also recommended that women avoid birth control pills during this same time period, as the increased estrogens can lead to hyperpigmentation. 


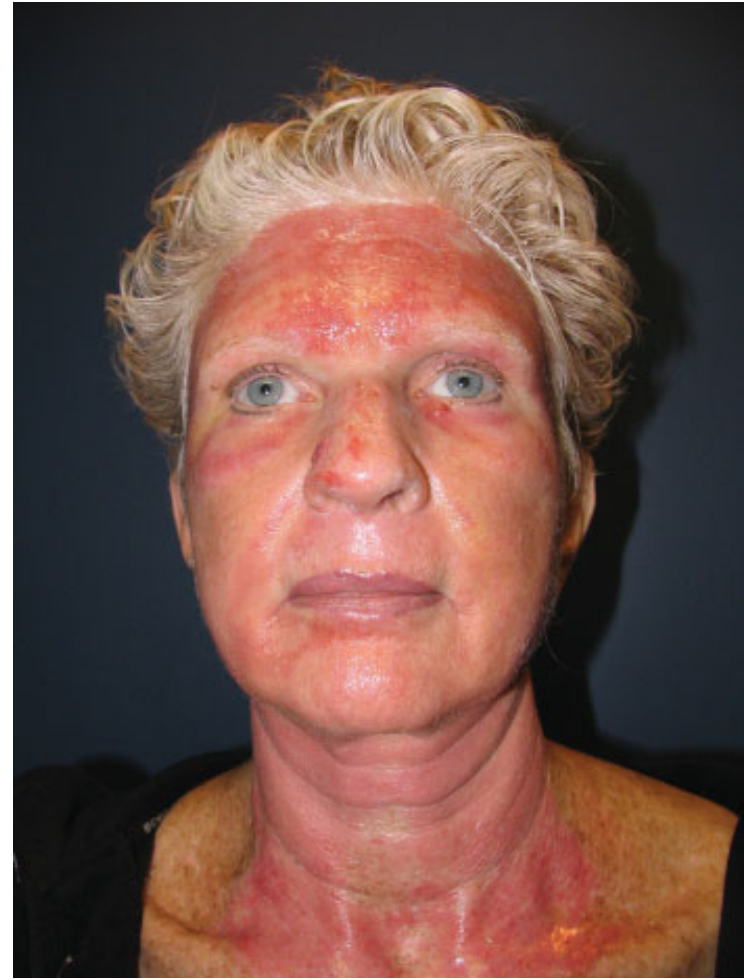

Fig. 3 Desquamation of the skin that takes place in the first 7 days following a phenol-croton oil peel.

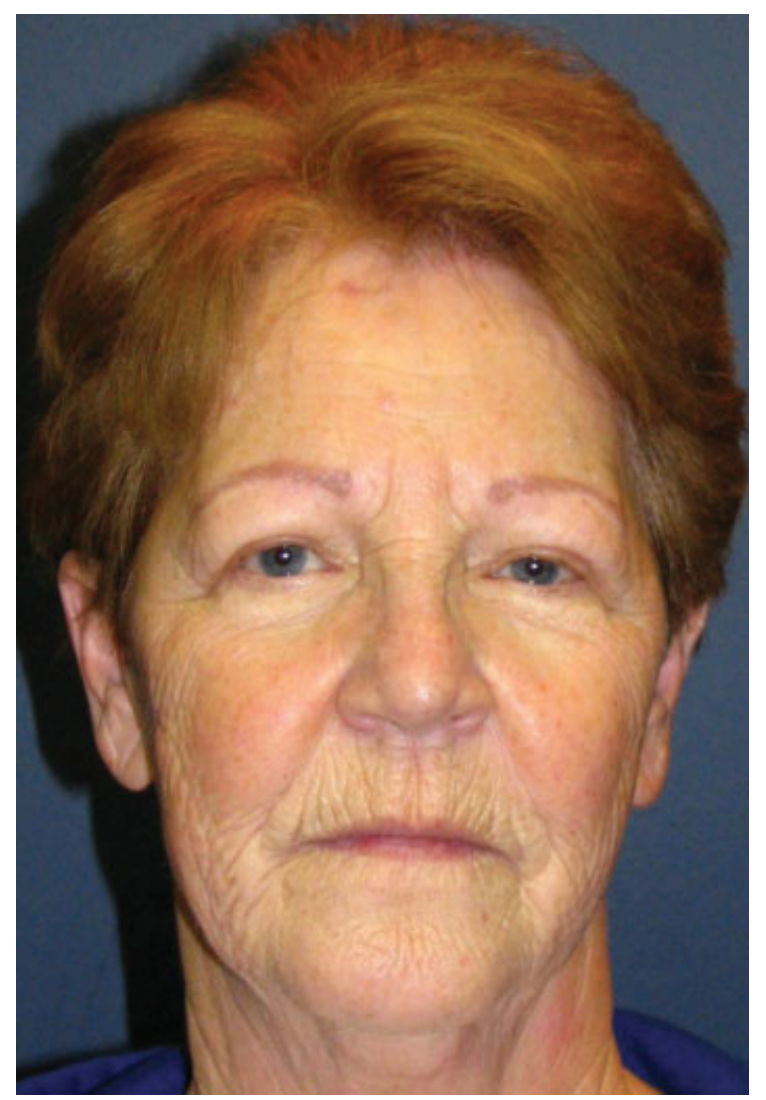

Fig. 4 Preoperative photograph of a patient with diffuse fine rhytids and photoaged skin.

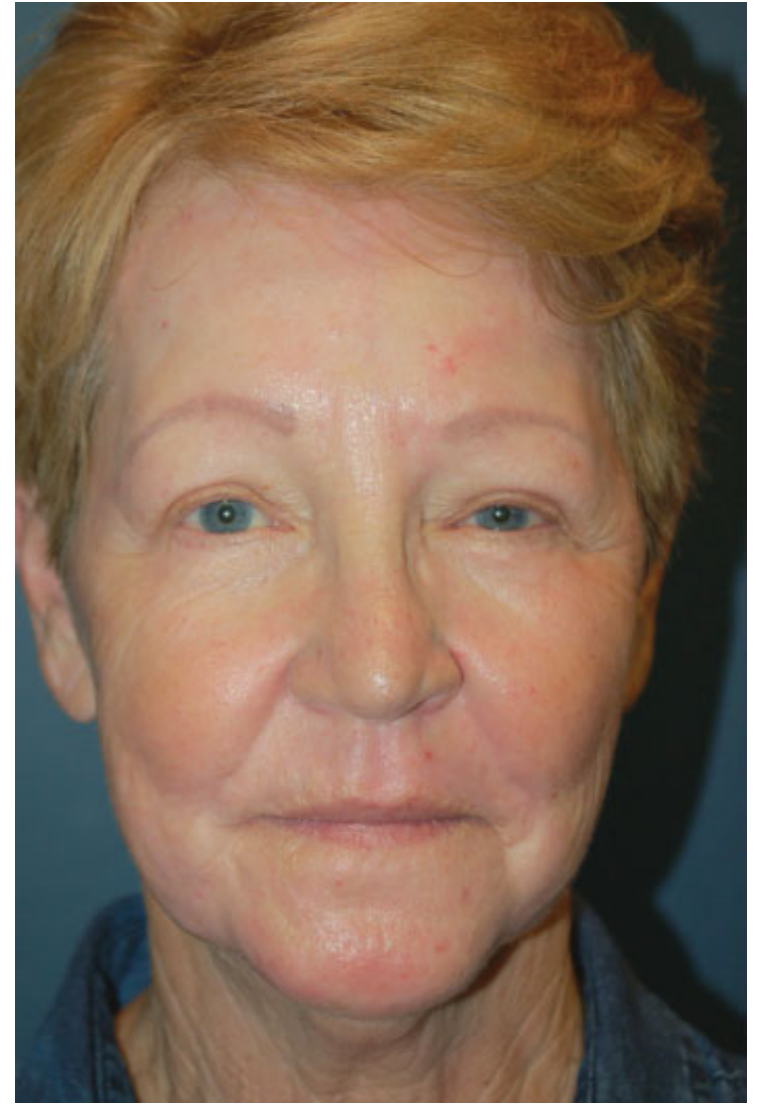

Fig. 5 Post phenol-croton oil peel photograph with reduction of the fine rhytids and treatment of the photoaged skin.

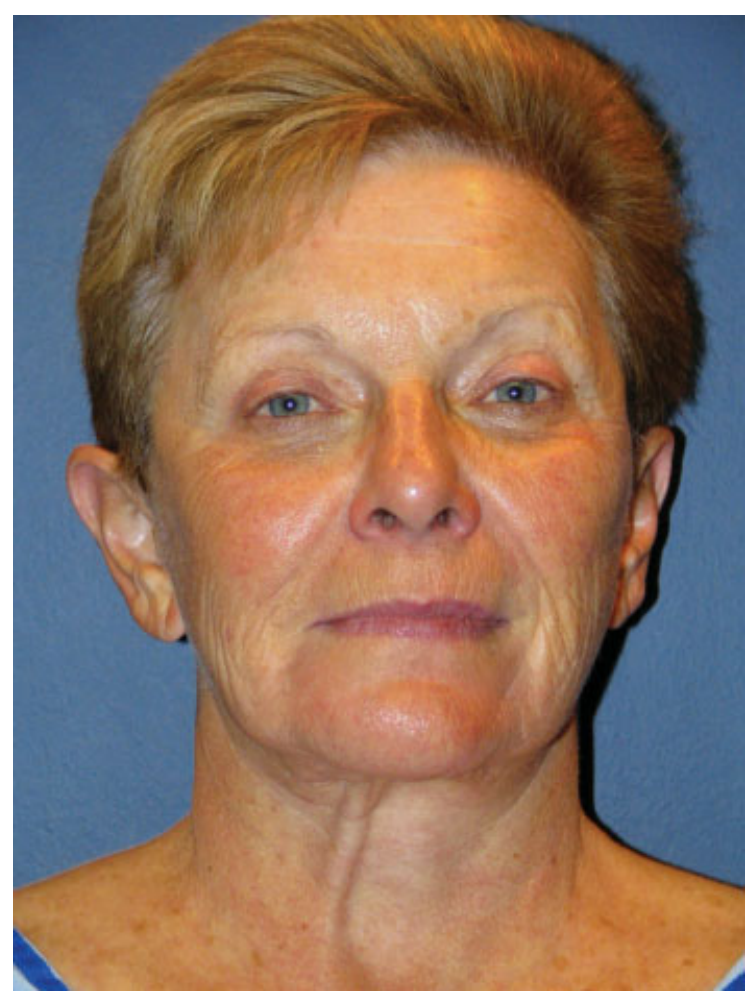

Fig. 6 Preoperative photograph of a patient with severe photodamage and moderate dyschromias and fine rhytids. 


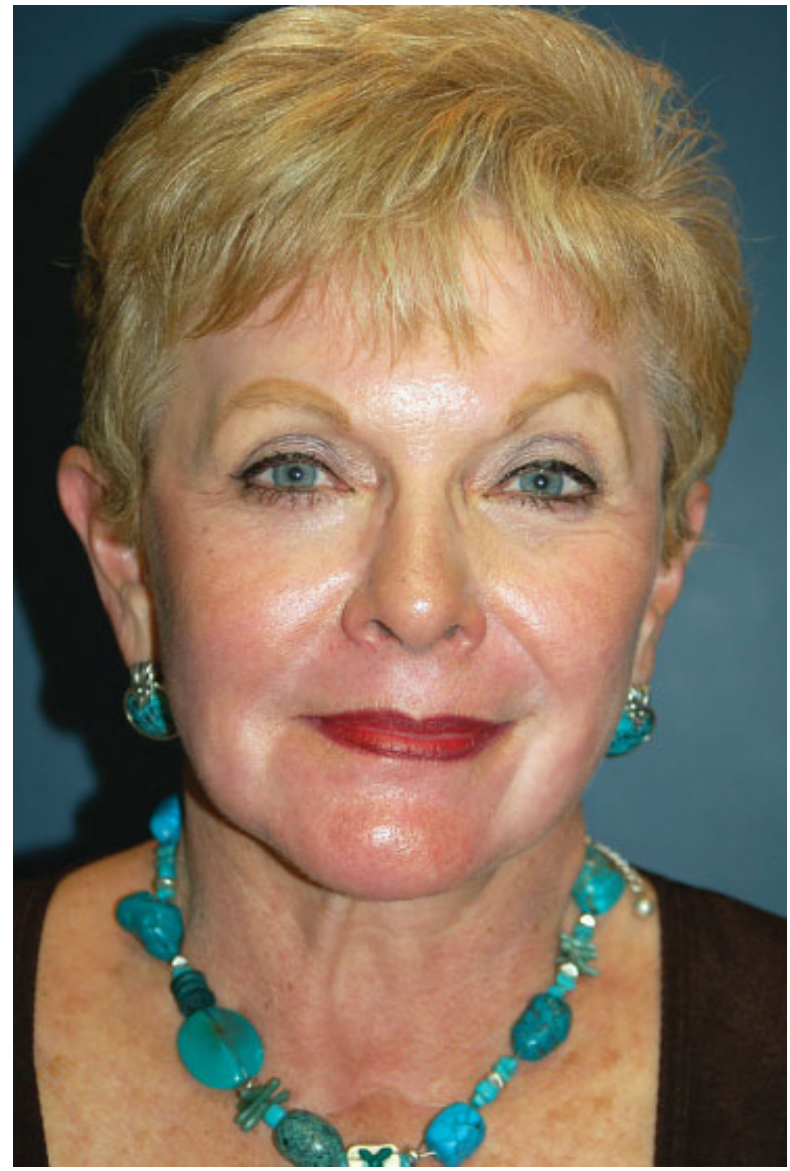

Fig. 7 Post phenol-croton oil photograph with treatment of the photodamage, dyschromias, and rhytids.

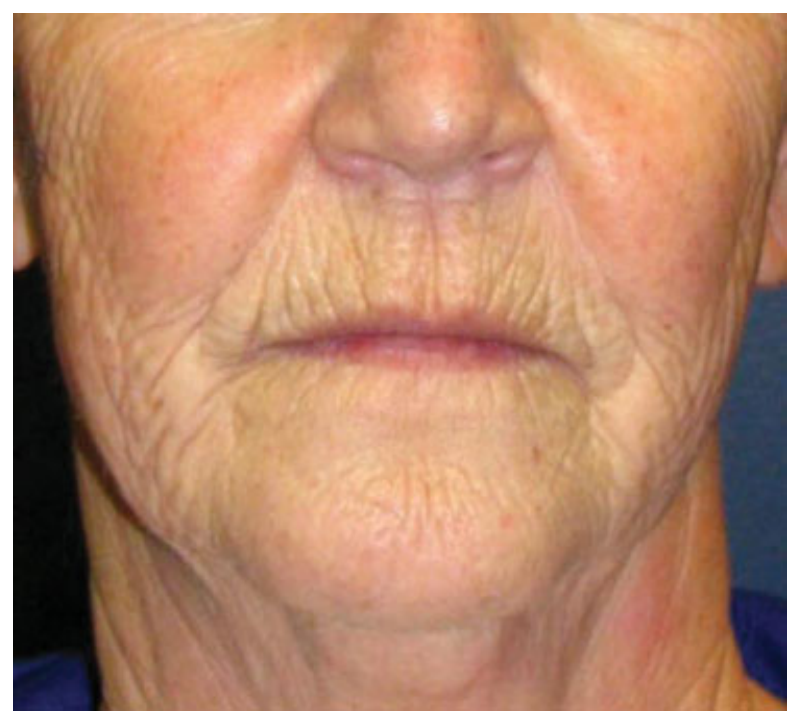

Fig. 8 Preoperative photograph of the perioral region with severe rhytids.

\section{Complications}

\section{Cardiac Arrhythmias}

Even in patients who have been properly selected and adequately hydrated before their chemical peel, a reversible cardiac arrhythmia can possibly occur. The common presenta-

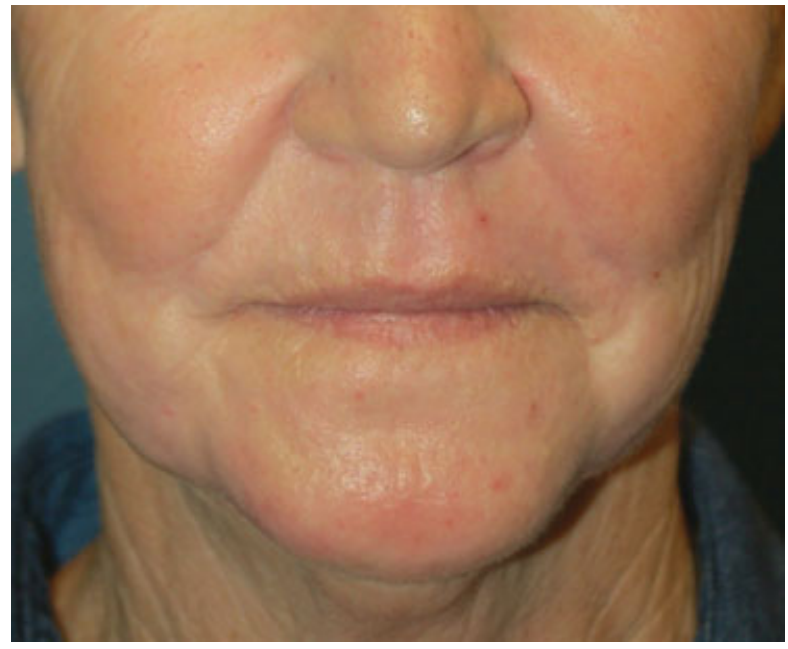

Fig. 9 Post phenol-croton oil peel photograph with treatment of perioral rhytids.

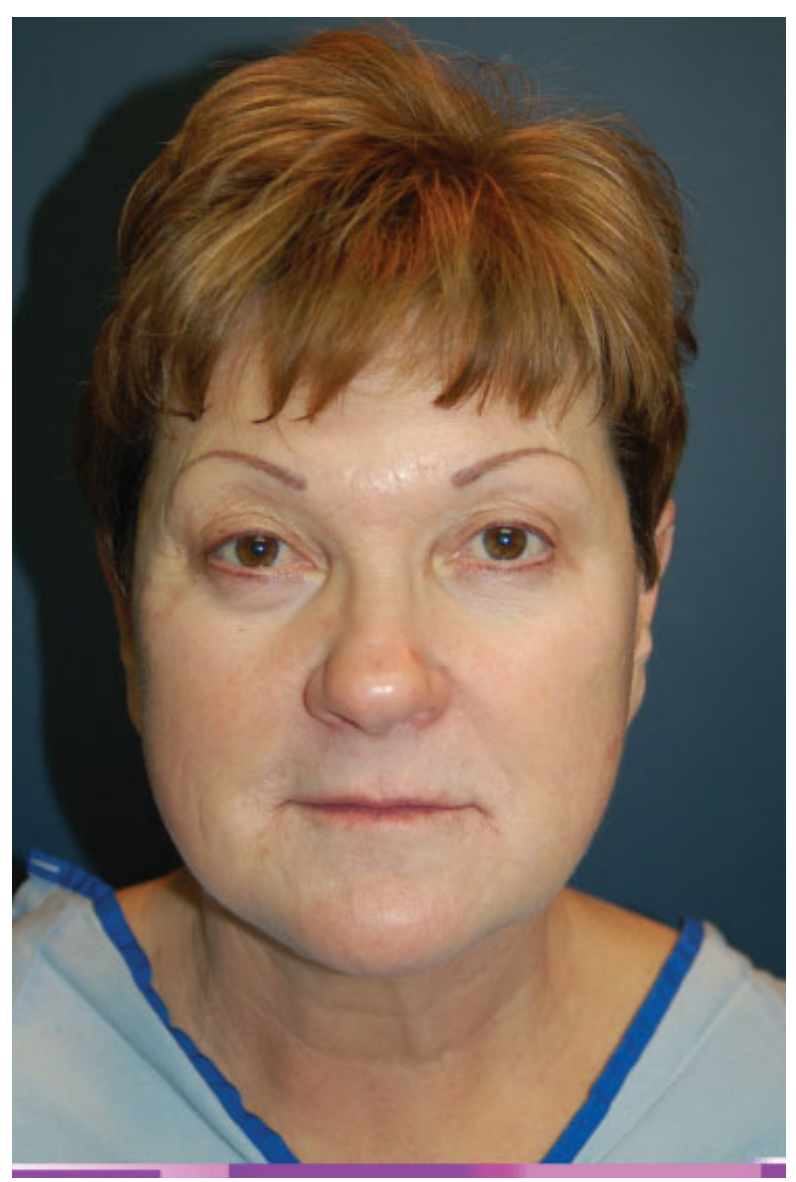

Fig. 10 Preoperative photograph of a patient with diffuse fine rhytids in the perioral, malar, and periorbital regions.

tion is a supraventricular tachycardia that occurs within 30 minutes of starting the peel and can evolve into paroxysmal atrial tachycardia, ventricular tachycardia, paroxysmal ventricular contractions, and, possibly, atrial fibrillations. The best way to manage any of these aforementioned progressive arrhythmias is to prevent them from occurring in the first place. As soon as a supraventricular tachycardia, or other 


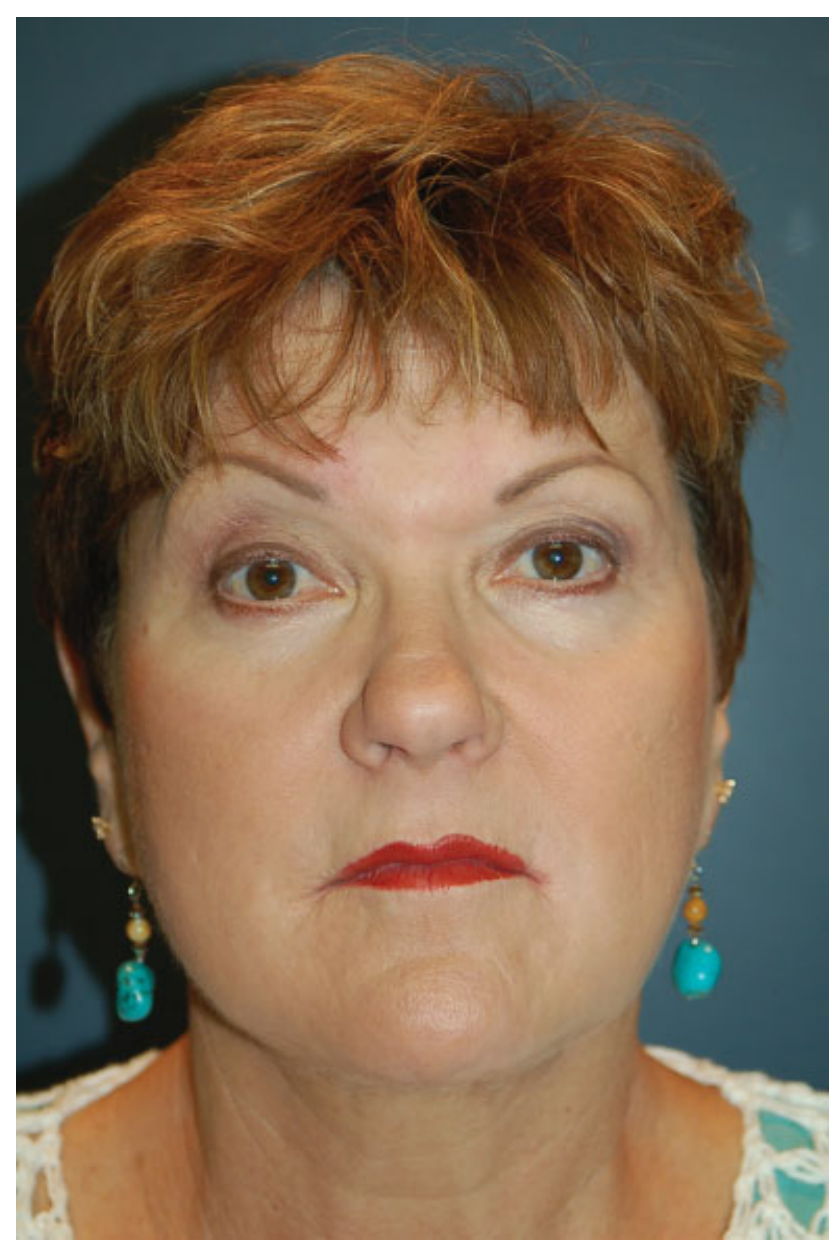

Fig. 11 Post phenol-croton oil photograph with treatment of diffuse fine rhytids.

irregular rhythm, is noted, the peel should be immediately paused and adequate hydration should continue. At this point, the rhythm should eventually return to normal sinus rhythm as the phenol is cleared. The phenol peel may proceed carefully with attention to the rhythm monitor once the rhythm has returned to normal. In the rare instance that the rhythm does not naturally return to a normal rhythm, proper medical procedures should be undertaken for that aberrant rhythm.

\section{Delayed Reepithelialization}

Any area of the face that does not fully reepithelialize within 10 days should be considered prolonged. ${ }^{18}$ This phenomenon is more common with deeper phenol peels (Baker formula) and TCA peels. It is important to rule out the presence of underlying infections or contact irritants and not merely dismiss prolonged healing times as coincidentally. The risk of scarring can rise precipitously if these areas are not checked daily and treated accordingly.

\section{Scarring}

The most likely areas for scarring to occur are in the upper lip or over areas with prominent underlying bone structure such as the mandible. Scarring most commonly is because of an overly deep peel or from inattentive postoperative care. Again, the risk of scarring is significantly elevated in isotretinoin users. The practitioner should check to confirm that the patient is clearly producing skin oils after the patient has stopped isotretinoin. Once developing, the scars can be treated with silicone sheeting coverings and intralesional corticosteroids injections (Kenalog $20 \mathrm{mg} / \mathrm{mL}$ ) every 2 to 3 weeks. It is important to exercise caution in the injections of steroids, as overinjection can lead to atrophy and skin depressions. As most scars will be erythematous, a flashlamp pulsed dye laser is helpful over multiple treatments.

\section{Infections}

In the case of a patient presenting with signs of cellulitis or infection, an appropriate antibiotic regimen should be immediately started and continued for a 7- to 10-day course. Similarly, herpetic viral infections can be problematic for a patient's natural recovery. In the case of a herpetic outbreak despite appropriate prophylactic dosing of antivirals, a course of valacyclovir, $1 \mathrm{~g}$ three times a day for 10 days, should be used.

\section{Postoperative Erythema}

Postoperative erythema after a peel is common in all peel patients and is not unusual for it to last longer than predicted. Hydrocortisone (2.5\%) lotion is commonly prescribed to aid in the resolution of this erythema. As this erythema is eventually subsiding in the weeks following the peel, some patients will develop PIH. The typical scenario for this is in a patient with excessive sun exposure following the peel or with Fitzpatrick skin types III and VI. This can be managed with a combination of $0.05 \%$ retinoic acid, $2.5 \%$ hydrocortisone cream, and $4 \%$ hydroquinone cream.

\section{Hypopigmentation}

One of the most severe complications of chemical peeling is hypopigmentation. This is most likely because of phenol's ability to eliminate melanocyte's ability to produce melanin. Hypopigmentation is much more readily noticeable when single facial subunits are peeled rather than the whole face. This complication was more common in the past when deeper peels such as the classic Baker formulation were used, as well as postoperative occlusive dressing applications. Hypopigmentation is unfortunately irreversible, and all patients who experience some level of this should be advised about the potential need for makeup usage.

\section{Conclusion}

Phenol-croton oil chemical peels have withstood the test of time and are still considered the standard against which other facial resurfacing procedures are judged. More recently, the widely expanded knowledge of croton oil, and its various concentrations, has yielded many options for specializing treatment for different facial subunits, skin 
types, thicknesses, and so on. A strong familiarity with the fundamentals and techniques of chemical peeling can result in predictable and excellent results.

\section{Conflicts of Interest}

The authors have no conflicts of interest to disclose.

\section{References}

1 Brody HJ. Complications of chemical peeling. J Dermatol Surg Oncol 1989;15(09):1010-1019

2 Nikalji N, Godse K, Sakhiya J, Patil S, Nadkarni N. Complications of medium depth and deep chemical peels. J Cutan Aesthet Surg 2012;5(04):254-260

3 Brody HJ. Complications of chemical resurfacing. Dermatol Clin 2001;19(03):427-438, vii-viii

$4 \mathrm{Kim} \mathrm{IH,} \mathrm{Kim} \mathrm{HK,} \mathrm{Kye} \mathrm{YC.} \mathrm{Effects} \mathrm{of} \mathrm{tretinoin} \mathrm{pretreatment} \mathrm{on} \mathrm{TCA}$ chemical peel in guinea pig skin. J Korean Med Sci 1996;11(04): 335-341

5 Popp C, Kligman AM, Stoudemayer TJ. Pretreatment of photoaged forearm skin with topical tretinoin accelerates healing of fullthickness wounds. Br J Dermatol 1995;132(01):46-53

6 Hevia O, Nemeth AJ, Taylor JR. Tretinoin accelerates healing after trichloroacetic acid chemical peel. Arch Dermatol 1991;127(05): 678-682
7 Yu RJ, Van Scott EJ. Alpha-hydroxy acids: science and therapeutic use. J Cosmet Dermatol 1994;1(Suppl 1):12

8 Van Scott EJ, Yu RJ. Alpha hydroxy acids: procedures for use in clinical practice. Cutis 1989;43(03):222-228

9 Lee KC, Wambier CG, Soon SL, et al , on behalf of the International Peeling Society(IPS). Basic chemical peeling-superficial and medium-depth peels. . J Am Acad Dermatol 2018 (e-pub ahead of print). doi:10.1016/ j.jaad.2018.10.079

10 Brody HJ. Variations and comparisons in medium-depth chemical peeling. J Dermatol Surg Oncol 1989;15(09):953-963

11 Dinner MI, Artz JS. Chemical peel-what's in the formula? Plast Reconstr Surg 1994;94(02):406-407

12 Brody HJ. Chemical Peeling and Resurfacing. St. Louis, MO: Mosby; 1997:109-110

13 Monheit GD. Advances in chemical peeling. Facial Plast Surg Clin North Am 1994;2:5-9

14 Herbig K, Trussler AP, Khosla RK, Rohrich RJ. Combination Jessner's solution and trichloroacetic acid chemical peel: technique and outcomes. Plast Reconstr Surg 2009;124(03):955-964

15 Coleman WP III, Futrell JM. The glycolic acid trichloroacetic acid peel. J Dermatol Surg Oncol 1994;20(01):76-80

16 Monheit GD. Medium-depth chemical peels. Dermatol Clin 2001; 19(03):413-425, vii

17 Hetter GP. An examination of the phenol-croton oil peel: Part III. The plastic surgeons' role. Plast Reconstr Surg 2000;105(02):752-763

18 Szachowicz EH, Wright WK. Delayed healing after full-face chemical peels. Facial Plast Surg 1989;6:8-13 